\title{
Precision Improvement of Power-Efficient Capacitive Senor Readout Circuit Using Multi-Nested Clocks
}

\author{
Longjie Zhong, Donglai Xu, Senior Member, IEEE, Xinquan Lai, Member, IEEE,
}

Yuheng Wang, Xinqing Liao, Zhongyuan Fang, and Yuanjin Zheng, Senior Member, IEEE

\begin{abstract}
This paper proposes a clock strategy to improve the precision of power-efficient readout circuit for capacitive sensor. To achieve high power efficiency, capacitive sensor such as MEMS accelerometer is designed with open-loop architecture rather than close-loop one. In the open-loop architecture, the capacitance variation of sensing element is limited to femto-farad level in order to overcome nonlinearity problem. However, due to this limitation, the signal charge from sensing element is weak and the interference charge due to coupling capacitance between clock wires and sensing electrodes becomes a significant issue. Therefore, split clock bus is employed to meet this challenge, but the split clock bus introduces the timing mismatch problem which causes charge injection sensitive to fabrication process and circuit operating voltage. As a result, the offset variation and nonlinearity of readout circuit will increase, and this will lead to reduction of precision. In this work, a clock scheme named "multi-nested clocks" is proposed to address the charge injection problem due to timing mismatch. The multi-nested clocks are demonstrated in a readout circuit fabricated using a $0.18-\mu \mathrm{m}$ BCD process. The measurement results show that compared to the readout circuit using traditional clock, the readout circuit using the multi-nested clocks reduces the equivalent input offset from $1.66 \mathrm{fF}$ to $0.25 \mathrm{fF}$, the offset variation from $1.4 \mathrm{fF}$ to $0.2 \mathrm{fF}$ and the nonlinearity from $5.5 \%$ to $0.9 \%$.
\end{abstract}

Index Terms-Capacitive sensor, MEMS, readout circuit, sensor interface, charge injection, high precision, nonlinearity, multi-nested clocks.

\section{INTRODUCTION}

M EMS (Micro-electromechanical Systems) capacitive sensor, especially accelerometer, is one of the most important large-scale medical data collectors in disease research such as cardiovascular diseases, diabetes and

This work was supported in part by the National Natural Science Foundation of China (NSFC) under Grant 61771363, and in part by National Research Foundation of Singapore under Grant No. NRF-CRP11-2012-01.

L. Zhong, X. Lai and Y. Wang are with the Institute of Electronic Computer-Aided Design, Xidian University, Xi'an 710071, China, and also with the Key Laboratory of High-Speed Circuit Design and Electromagnetic Compatibility, Ministry of Education, Xi'an 710071, China (e-mail: zhonglongjie4213@126.com; xqlai@mail.xidian.edu.cn).

D. Xu is with the School of Electrical and Electronic Engineering, Wuhan Polytechnic University, Wuhan 430048, China and the School of Science and Engineering, Teesside University, Middlesbrough TS1 3BA, U.K. (email: d.xu@ tees.ac.uk).

Y. Zheng, X. Liao and Z. Fang are with VIRTUS, School of Electrical and Electronics Engineering, Nanyang Technological University, Singapore (e-mail: yjzheng@ntu.edu.sg). psychosis [1]-[3]. It plays an important role in continuous physical activity monitoring and health care applications [4][5]. In these applications, the sensors are powered by battery, thus high energy efficiency is required to extend battery life. As a result, the MEMS accelerometers for wearable monitoring devices are designed with open-loop architecture rather than close-loop one to achieve low power consumption [6]. In the open-loop architecture, the nonlinearity of the mechanical sensing element significantly increases with the increase of the capacitance variation of the sensing element. Therefore, the capacitance variation needs to be limited to femto-farad level to suppress the nonlinearity to an acceptable level. However, the fact that the femto-farad level capacitance variation of sensing element is much lower than parasitic capacitance of sensing electrodes which is at pico-farad-level results in significant deterioration of gain error [7]. The gain error deterioration can be alleviated by the "Oversampling Successive Approximation (OSA) Readout technique" [7][8].

Compared to traditional readout circuit [9]-[12], the OSA based readout circuit shows advantages in gain accuracy and power efficiency. However, the OSA based readout circuit is sensitive to interference charge due to the coupling capacitance between clock wires and sensing electrodes, which introduces such a significant offset that it can easily overwhelm the signal amd saturate the readout circuit. Traditionally, the correlated double sampling (CDS) technique is employed to cancel the offset of capacitive readout circuit introduced by amplifier [11]-[13]. But the offset introduced by the interference charge from clock wires can hardly be sampled and cancelled with the CDS technique, as it's difficult to predicate which clock wire the interference charge will come from and when the interference charge will come. To avoid this unpredictable interference charge from clock wires, this work employs a layout-level solution named "split clock bus", in which the clock wires are symmetrically routed outside circuit blocks rather than inside circuit blocks to eliminate coupling effect in the physical layout. Although the high precision auto-calibration technique can be employed to reduce the offset of readout circuit resulted from interference charge to sub-fF level [14]-[16], the split clock bus can reduce the offset without additional redundancy calibration circuit. However, this split clock bus has a drawback that it causes "timing mismatch" which introduces charge injection varying with fabrication 
process. This variable charge injection will result in offset variation and nonlinearity in the readout circuit. In this paper, a clock scheme named "multi-nested clocks" is proposed to solve this problem. Compared with the traditional charge injection reduction methods such as the dummy switch [17][18], the injection-nulling switch [19], the low swing driver [20] and the multi-frequency operation [21], the proposed method can not only reduce the charge injection, but also reduce the charge injection variation, thus improve the precision of readout circuit.

The rest of the paper is organized as follows. In Section II, the phenomenon of timing mismatch is illustrated in detail. In Section III, charge injection of readout circuit are analyzed with four models. Multi-nested clocks for minimizing the charge injection and charge injection variation of each model are described. In Section IV, the multi-nested clocks are demonstrated in the OSA based readout circuit and the measurement results are presented. The conclusions are then drawn in Section V.

\section{TIMING MISMATCH}

\section{A. Split clock wire and timing mismatch}

The readout circuit based on the OSA technique shown in Fig. 1 is composed of the common-mode charge controller (CMC) to absorb the unwanted common-mode charge produced by the sensor and the capacitance-to-voltage convertor (CVC) to convert the capacitance difference $\left(C_{S 1}-C_{S 2}\right)$ of the sensing element to the differential output voltage $\left(V_{O_{+}}-V_{O_{-}}\right)$. The capacitors $C_{P 1}$ and $C_{P 2}$ represent the parasitic capacitance between the sensing electrodes (IN+ and IN-) and the ground. The capacitors $C_{P 3}$ and $C_{P 4}$ represent the coupling capacitance between the sensing electrodes and the interference voltage source $V_{I N T}$. The capacitor $C_{L}$ and the switches in the small dash box represent the switched-capacitor loads such as the switched-capacitor common-mode feedback network (SC-CMFB) used in the readout circuit [22]. The ideal output of the readout circuit is in proportion to the sensing element's capacitance difference,

$$
V_{O}=V_{O+}-V_{O-}=\frac{V_{R}}{C_{i}}\left(C_{S 1}-C_{S 2}\right)
$$

where $V_{R}$ is the reference voltage, $C_{i}$ is the integration capacitor. In this paper, only the charge injection related issues resulted from timing mismatch are discussed, as other properties of this readout circuit were explained in [7].

The most interference charge occurs in the sensing electrodes IN+ and IN- due to the coupling capacitances $C_{P 3}$ and $C_{P 4}$. Taking these coupling capacitances into consideration, the equation (1) becomes,

$$
V_{O}=\frac{V_{R}}{C_{i}}\left(C_{S 1}-C_{S 2}\right)+\frac{V_{I N T}}{C_{i}}\left(C_{P 3}-C_{P 4}\right)
$$

According to the typical 3-axis MEMS accelerometer from STMicroeletronics [23][24], the typical variation of the sensing element's capacitance difference $\left(C_{S 1}-C_{S 2}\right)$ is $1 \mathrm{fF}$ when $1 \mathrm{~g}$ acceleration is applied. The reference voltage $V_{R}$ is $0.9 \mathrm{~V}$. The

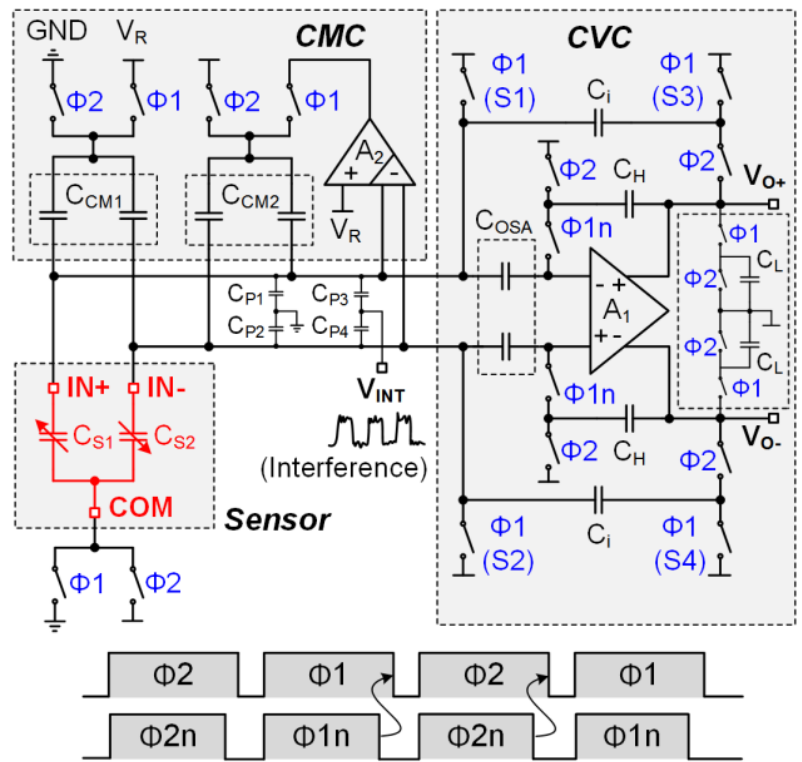

Fig. 1. Readout circuit based on the OSA technique.

interference source $V_{I N T}$ usually stems from the clock wires and it is at a similar voltage level to VDD $(1.8 \mathrm{~V})$. As a result, if the mismatch of the coupling capacitances $C_{P 3}$ and $C_{P 4}$ reaches $0.5 \mathrm{fF}$, the interference charge would be large enough to overwhelm the signal charge.

Practically, it is difficult to match the coupling capacitances in physical layout in CMOS process to a sub-fF level. Thus, the interference charge is inevitable and it is undeterminable as well. Although calibration method can be employed to reduce the mismatch, additional design will be needed and the noise from $V_{I N T}$ is still inevitable. A better way to deal with the interference charge caused by the coupling capacitances $C_{P 3}$ and $C_{P 4}$ is use of the "split clock bus" rather than the "concentrated clock bus" in the physical layout, as shown in Fig. 2 . With the split clock bus, all the clock wires are routed outside the circuit blocks to avoid any undesirable coupling with the sensing electrodes. The clock wire is "split" because the circuit structure is fully differential. For example, the bus-1A drives the switches in one differential circuit branch (the switches S1 and S3 in Fig. 2(b)), and the bus-1B drives the switches in the other differential circuit branch (the switches S2 and S4 in Fig. 2(b)). In this way, the clock wires will not have undesirable coupling with the wires inside the blocks.

However, the split clock bus causes a new problem "timing mismatch" which includes delay mismatch and slew-rate mismatch, as shown in Fig. 3. This is because the two branches of the split bus (e.g., bus-1 A and bus-1B in Fig. 2(b)) are on two different physical routes from which any mismatch of coupling capacitances to the nearby ground/power/clock wires will lead to the timing mismatch between clocks the driving two differential circuit branches. This timing mismatch then introduces the charge injection which varies with fabrication process and is therefore difficult for traditional charge injection reduction methods to deal with.

\section{B. Charge injection models of timing mismatch}




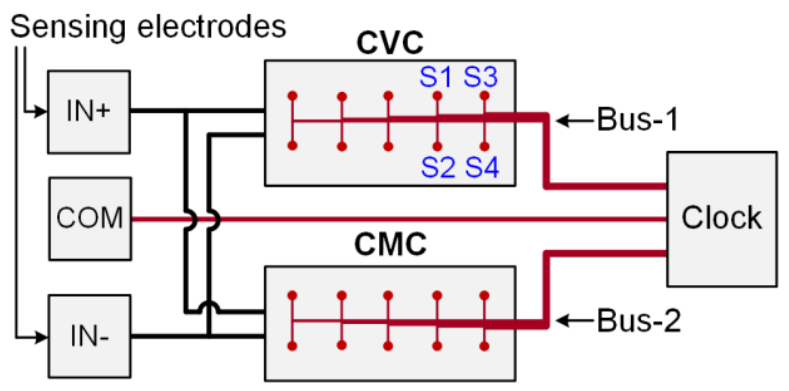

(a)

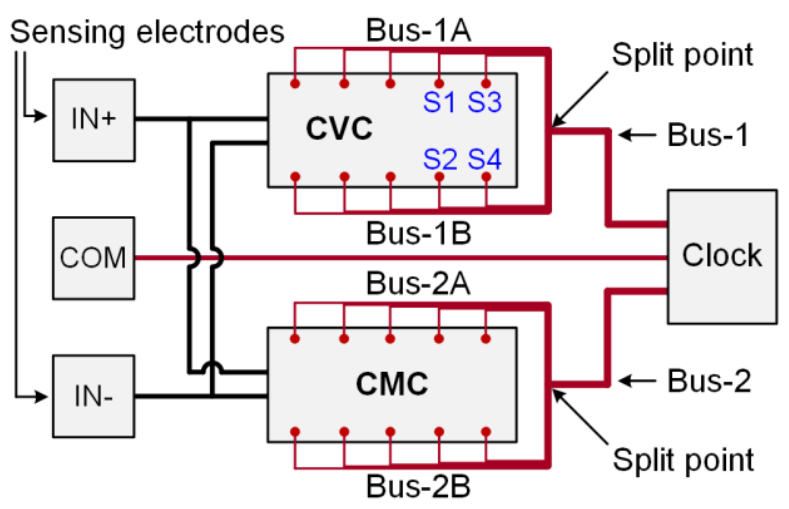

(b)

Fig. 2. Layout consideration of clock routing for the readout circuit. (a) Concentrated clock bus. (b) Split clock bus.

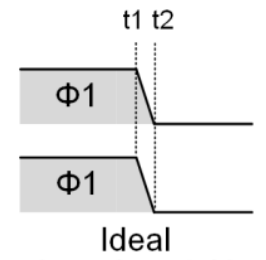

(no mismatch)
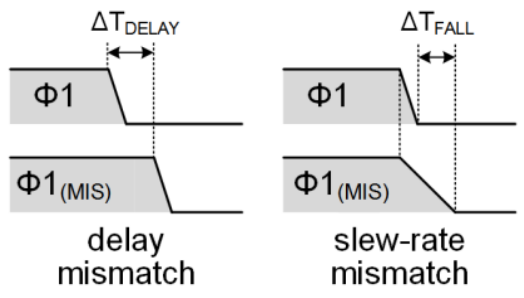

Fig. 3. Timing mismatch between the clocks driving two differential branches in the split clock bus.

The charge injections resulted from the timing mismatch can be modelled as follows. There are four types of charge injection models as shown in Fig. 4, which are derived from the schematic in Fig. 1(a) with the consideration of the charge injections to all the capacitors. Although these models are derived from the OSA based readout circuit, they are also effective to predicate the charge injections in the conventional switched-capacitor readout circuit.

The Type-I model shown in Fig. 4(a) is the classical charge injection model which consists of one switch and one capacitor [17][18]. This model is only suitable for parasitic capacitance, such as the capacitors $C_{P 1}$ and $C_{P 2}$ in Fig. 1, one of whose terminals is permanently connected to DC sources. The Type-II model shown in Fig. 4(b) is suitable to describe the charge injection to those capacitors, both of whose terminals are connected to switches, such as the capacitor $C_{i}$. The Type-III model shown in Fig. 4(c) is used to describe the charge injection to the two capacitors in different circuit branches but with a common node, such as the capacitors $C_{S 1}$ and $C_{S 2}$. The capacitors $C_{S 1}$ and $C_{S 2}$ are also known as "capacitive half-bridge" [25]. The Type-IV model shown in Fig. 4(d)

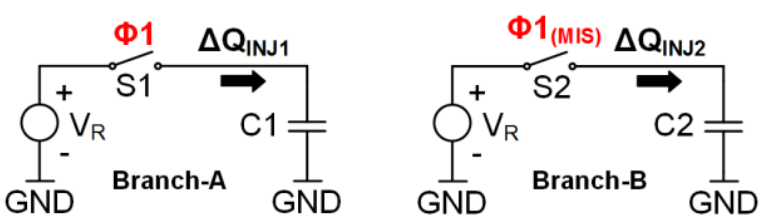

(a)

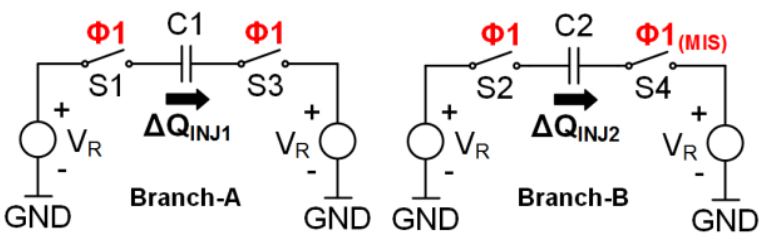

(b)

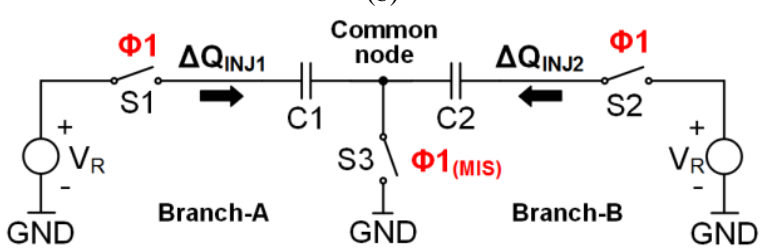

(c)

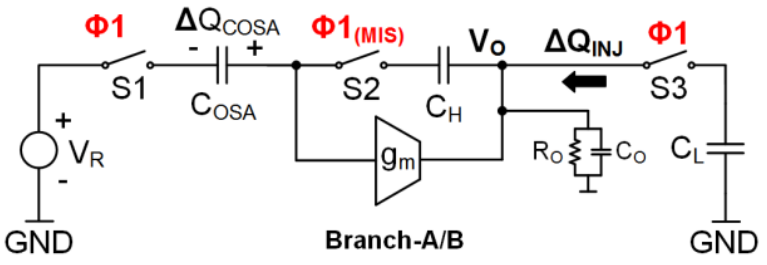

(d)

Fig. 4. Charge injection models. (a) Type-I model for injections to parasitic capacitors (e.g., $C_{P}$ ). (b) Type-II model for injections to operational capacitors (e.g., $C_{i}$ ). (c) Type-III model for injections to capacitive half-bridge (e.g., $C_{S 1} \& C_{S 2}, C_{C M 1}$ and $C_{C M 2}$ ). (d) Type-IV model for kickback charge injections to capacitors in the feedback network (e.g., $C_{H}$ and $C_{O S A}$ ).

describes the kickback charge injection to the capacitors in the feedback network, such as the capacitors $C_{H}$ and $C_{O S A}$. This type of charge injection is a result of voltage spike of the output terminals [26].

These models are analyzed only in the phase $\Phi 1$. This is because the readout circuit's output is affected by the charge injection only generated in the phase $\Phi 1$.

\section{REDUCTION OF VARIATION OF CHARGE INJECTION}

\section{A. Analysis of Type-I model}

Traditionally, the reduction of the charge injection in Type-I model can be achieved by using the dummy switches [18], as shown in Fig. 5(a). Ideally, the amount of the charge compensated by the dummy switches is the same as the amount of the charge injected by the main switch, therefore completely compensating the charge injection. However, this method will lose its effectiveness if any of the following three parameters varies: the reference voltage $V_{R}$ (it is used as bias voltage), the load capacitor $\mathrm{C} 1$ and the slew-rate of the clock. This is explained as follows and the solutions are given thereafter.

When biased with different voltages, the main switch will inject different amount of charge [17]. The value of injected charge is the smallest when the bias voltage is near half of the power supply voltage (i.e., $0.8 \mathrm{~V}-1.0 \mathrm{~V}$ ). This is because the 


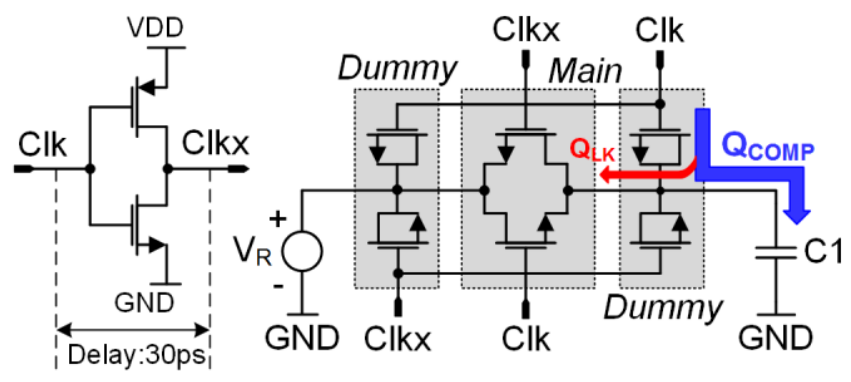

(a)

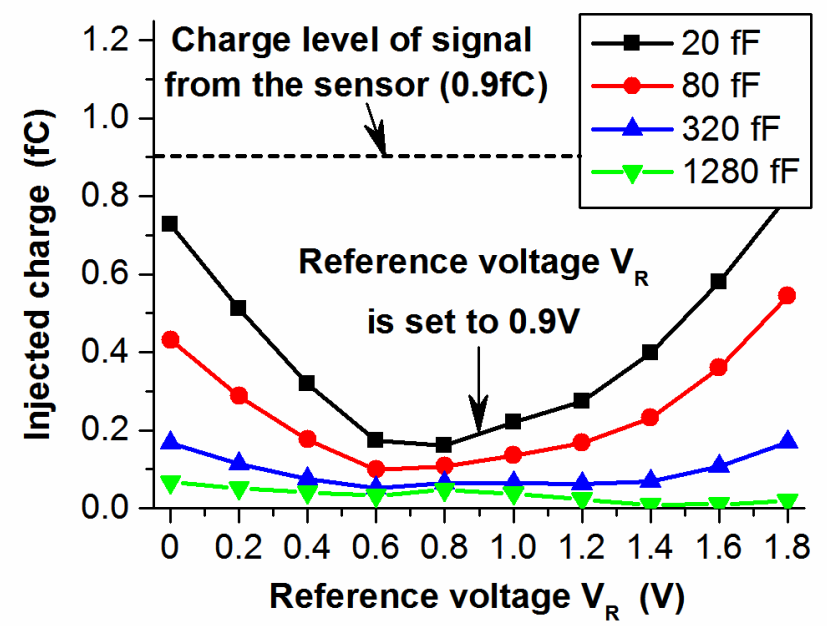

(b)

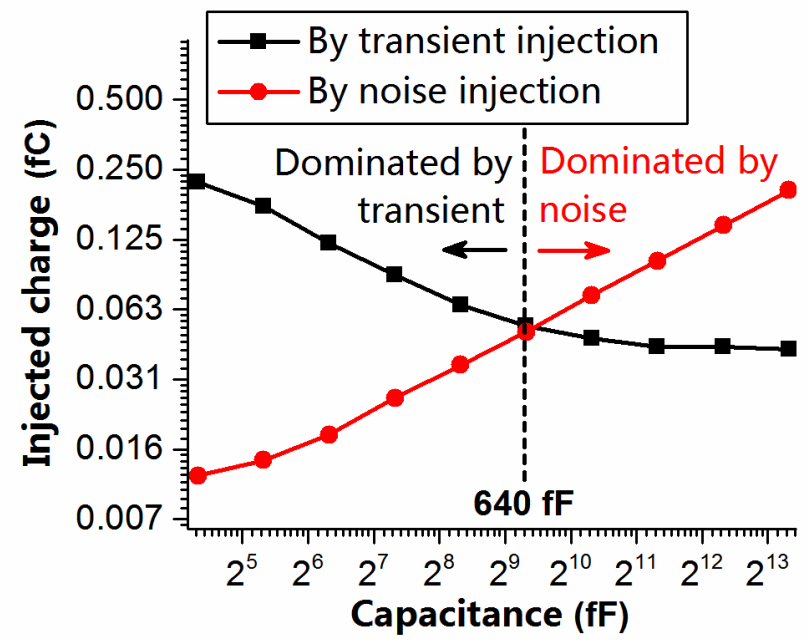

(c)

Fig. 5. Basic charge injection properties of the traditional switch. (a) Schematic of the traditional switch configured as Type-I model. (b) Injected charge versus bias voltage $V_{R}$. (c) Injected charge versus capacitance of $C 1$.

charge injections from P-type MOSFET and N-type MOSFET of the main switch cancel each other well when the main switch is biased in such a way. Therefore, all the reference voltages in the readout circuit are set to be $0.9 \mathrm{~V}$ to minimize charge injection, as shown in Fig. 5(b).

The capacitance of $\mathrm{C} 1$ affects amount of the injected charge from the traditional switch to the capacitor $\mathrm{C} 1$ in two aspects: the transient charge injection due to clock activities and the thermal noise charge injection due to on-resistance of transistor. The smaller the capacitance of $\mathrm{C} 1$ is, the larger the transient charge injection will be. In Fig. 5(a), the falling-edge of the clock Clk is 30ps earlier than that of the inverse clock Clkx. Thus, the compensation charge $Q_{\text {СOMP }}$ from the dummy switches is injected before the main switch is completely shut down. As a result, part of the compensation charge $Q_{\text {СоMP }}$ is leaked via the main switch and the compensation is less effective. Amount of the leaked compensation charge $Q_{L K}$ is adversely proportional to the value of $\mathrm{C} 1$. Thus, small value of C1 will lead to ineffective compensation and high transient charge injection. On the other hand, the larger the capacitance of $\mathrm{C} 1$ is, the larger the thermal noise charge injection will be. According to equipartition theorem, the thermal noise charge from the transistors sampled by the capacitor $\mathrm{C} 1$ increases with the increase of the capacitance value of $\mathrm{C} 1$ [27].

The transistor-level simulation of the Type-I model is shown in Fig. 5(c). The channel widths/lengths of P-type MOSFET in the main switch, P-type MOSFET in the dummy switches, $\mathrm{N}$-type MOSFET in the main switch and N-type MOSFET in the dummy switches are $2 \mu \mathrm{m} / 200 \mathrm{~nm}, 1 \mu \mathrm{m} / 200 \mathrm{~nm}, 1 \mu \mathrm{m} / 200 \mathrm{~nm}$ and $500 \mathrm{~nm} / 200 \mathrm{~nm}$, respectively. These dimensions are also used in the remaining part of this paper. It is clear from Fig. 5(c) that for the capacitor $\mathrm{C} 1$ with a large capacitance (significantly larger than 640fF), it is essential to reduce the thermal noise charge injection as the overall injected charge is dominated by the thermal noise charge injection. For $\mathrm{C} 1$ with a small capacitance (significantly less than 640fF), it is important to reduce the transient charge injection as the overall injected charge is dominated by the transient charge injection. This work focuses on reducing the transient charge injection, as the sensing element capacitance is typically smaller than $640 \mathrm{fF}$ and the thermal noise charge injection is not dominant.

The mismatch variation of the slew-rate or slope of the clock Clk makes the charge injection of traditional switch variable. This was explained in detail in [17]. In this paper, a switch named as "local slew-rate controlling (LSC) switch" shown in Fig. 6(a) is employed to reduce the variation of the charge injection. As can be seen in the figure, an inverter chain composed of $\mathrm{N}$ inverters is added to the traditional switch to reduce the effect of slew-rate mismatch on charge injection. The transistor-level simulation results in Fig. 6(b) show that with the traditional switch, the amount of injected charge varies significantly with change of the slew-rate mismatch. However, with the LSC switch, the variation of charge injection is significantly reduced. With $\mathrm{N}=2$ and 4 , the variation is reduced from $336 \%$ per decade to $87.5 \%$ per decade and $5 \%$ per decade, respectively. It is unnecessary to make $\mathrm{N}$ larger than 4 , as the variation is already negligible (5\% per decade) when $\mathrm{N}=4$ and the additional delay due to large $\mathrm{N}$ can be detrimental to the reduction of the delay mismatch which is to be explained in the next section.

In summary, the techniques to deal with charge injection and charge injection variation are as follows. Firstly, reference voltage is set to be $0.9 \mathrm{~V}$ for the least charge injection. Secondly, LSC switch is used to reduce the variation of charge injection, which is caused by variation of slew-rate mismatch. It is worth noting that although these techniques are derived from Type-I model, they are also applicable to the other three models. 


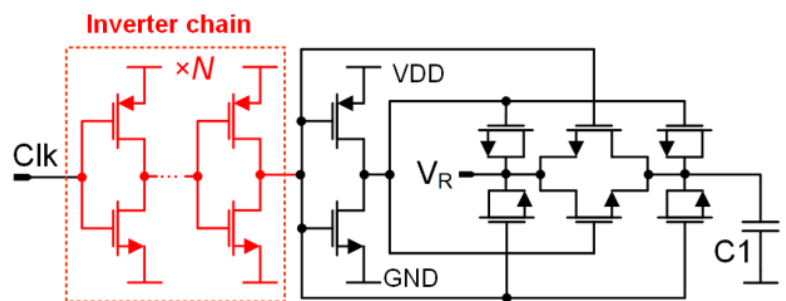

(a)

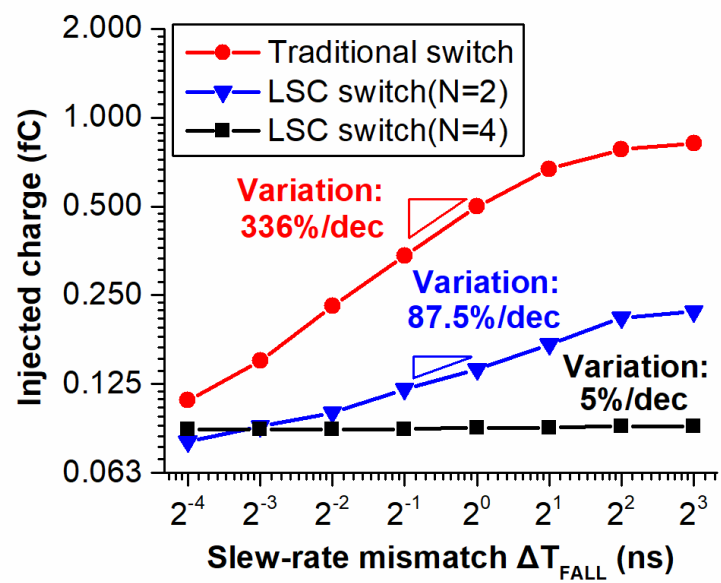

(b)

Fig. 6. Charge injection properties of LSC switch. (a) Schematic of LSC switch configured as Type-I model. (b) Charge injection with different slew-rate mismatch. The value of the capacitor $\mathrm{C} 1$ is $100 \mathrm{fF}$.

\section{B. Analysis of Type-II model}

Delay mismatch is the main consideration of Type-II model shown in Fig. 4(b) as the slew-rate mismatch can be solved by employing the LSC switches. The charge injection of the model is explained by the equivalent circuit in Fig. 7(a). The switches $\mathrm{S} 2$ and $\mathrm{S} 4$ are modeled as the variable resistors $R_{S 2}$ and $R_{S 4}$, respectively. The resistance of theses variable resistors varies with the delay mismatch $\Delta T_{D E L A Y}$. If $\Delta T_{D E L A Y}=0$, the switches $\mathrm{S} 2$ and $\mathrm{S} 4$ shut down simultaneously and $\mathrm{R}_{\mathrm{S} 2}$ is equal to $\mathrm{R}_{\mathrm{S} 4}$. As a result, the injected compensation charges $Q_{C O M P 1}$ from $\mathrm{S} 2$ and $Q_{C O M P 2}$ from $\mathrm{S} 4$ are equal and the total charge injected to $\mathrm{C} 2$ is zero. If $\Delta T_{D E L A Y}>0$, the switch $\mathrm{S} 2$ shuts down earlier than the switch $\mathrm{S} 4$ does and the resistance of $R_{S 2}$ is larger than that of $R_{S 4}$. Thus, the charge $Q_{C O M P 2}$ from S4 is leaked $\left(Q_{L K 2}\right)$ and the charge injected to $\mathrm{C} 2$ is not zero and it is dominated by the charge $Q_{C O M P 1}$. If $\Delta T_{D E L A Y}<0$, the charge injected to $\mathrm{C} 2$ is not zero either and it is dominated by $Q_{\text {COMP2 }}$.

The transistor-level simulation results in Fig. 7(b) show that the maximum variation of the charge injection caused by the delay mismatch can reach $0.8 \mathrm{fC}$, i.e., $89 \%$ of the signal charge (0.9fC) in Fig. 5(b). The variation of charge injection decreases if the variation of delay mismatch $\triangle T_{D E L A Y}$ is not in the range of $\Delta T_{V P 1}$ whose value is about 8 times of the falling time $T_{F A L L}$ of clocks. Thus, if the two clocks with a delay offset $\Delta T_{O F F S E T}$ larger than $\Delta T_{V P 1}$ (such as the clocks $\Phi 1$ and $\Phi 1 \mathrm{n}$ shown in Fig. 8) are used to drive S2 and S4, the charge injection variation problem in Type-II model can be minimized.

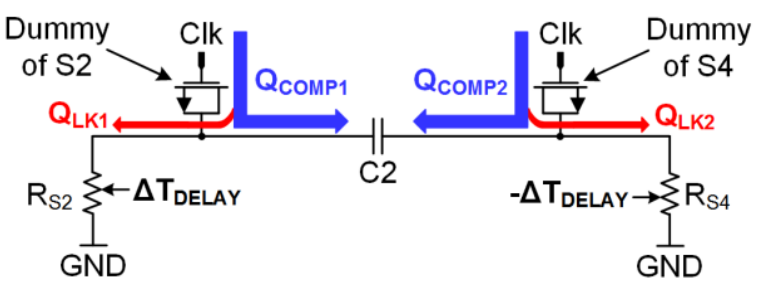

(a)

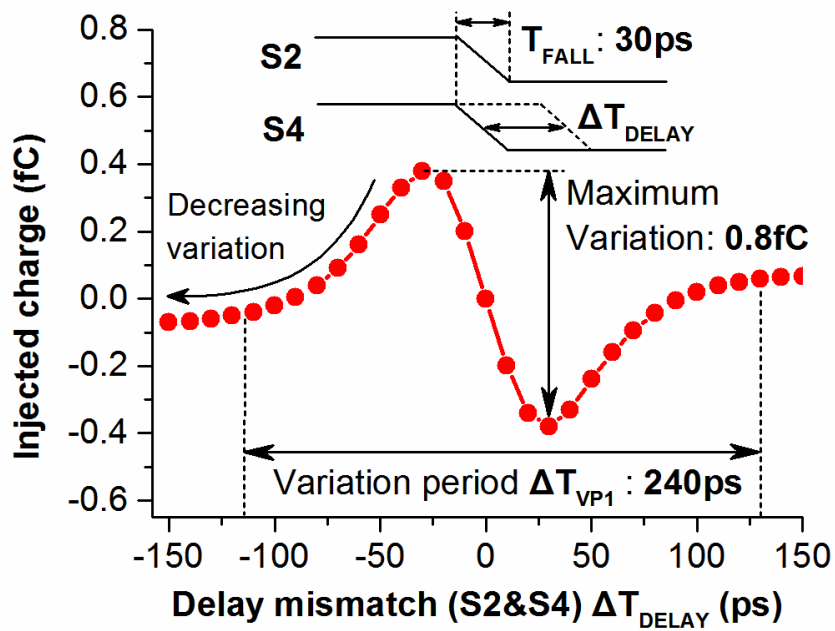

(b)

Fig. 7. Charge injection properties of Type-II model. (a) Equivalent circuit of Type-II model. (b) Transistor-level simulation results.

\section{Analysis of Type-III model}

The major consideration of Type-III model shown in Fig. 4(c) is the delay of the switch S3. If S3 switches off earlier than the switches S1 and S2 do, the common node of the capacitors C1 and C2 floats, as shown on the left in Fig. 9(a). So any charge injected to $\mathrm{C} 1$ will also be injected to $\mathrm{C} 2$ via the floating common node, but in opposite direction. Thus, the charge $Q_{I N J}$ injected from the switches $\mathrm{S} 1$ and $\mathrm{S} 2$ to the capacitors $\mathrm{C} 1$ and $\mathrm{C} 2$ is always differential charge injection.

If S3 switches off later than the switches $\mathrm{S} 1$ and $\mathrm{S} 2$ do, the common node of the capacitors $\mathrm{C} 1$ and $\mathrm{C} 2$ is grounded via $R_{S 3}$, as shown on the right in Fig. 9(a). The resistor $R_{S 3}$ is the turn-on resistance of the switch $\mathrm{S} 3$. In this mode, the charge injected from the switch $\mathrm{S} 1$ flows to the ground GND via $R_{S 3}$ rather than flowing into $\mathrm{C} 2$, so does the charge injected from the switch $\mathrm{S} 2$. As a result, the charges $\left(Q_{I N J 1}\right.$ and $\left.Q_{I N J 2}\right)$ injected to the capacitors $\mathrm{C} 1$ and $\mathrm{C} 2$ are in the same direction and they are common-mode charge injection.

The above analysis indicates that by delaying the switch-off time of the switch S3, it can prevent injecting differential charge to the capacitors $\mathrm{C} 1$ and $\mathrm{C} 2$, therefore minimizing differential charge injection in Type-III model. As shown in the transistor-level simulation results in Fig. 9(b), when S3 switches off 10ns later than $\mathrm{S} 1$ and $\mathrm{S} 2$ do, the differential charge injected to the capacitors $\mathrm{C} 1$ and $\mathrm{C} 2(\Delta \mathrm{Q} 1)$ is reduced by 18 times compared to the differential charge $\Delta \mathrm{Q} 2$ where $\mathrm{S} 3$ switches off 10ns earlier than S1 and S2 do. 


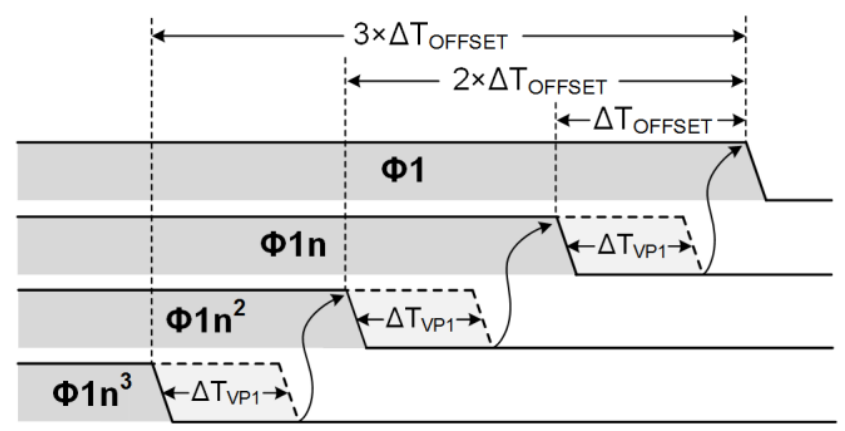

Fig. 8. Clock arrangement for reducing the variation of charge injection.

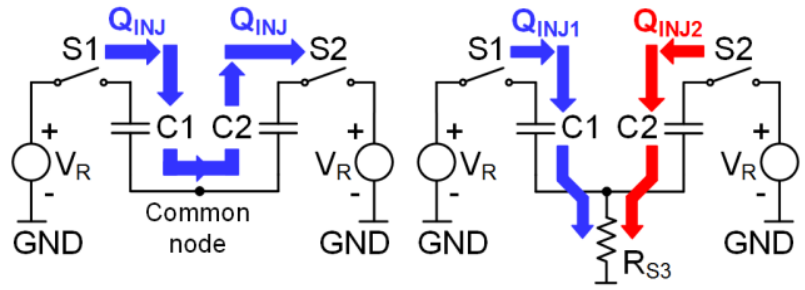

(a)

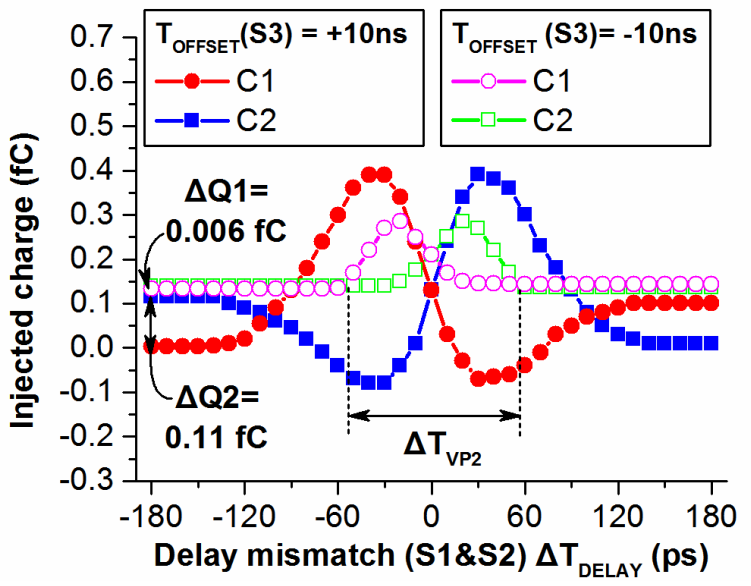

(b)

Fig. 9. Charge injection properties of Type-III model. (a) Equivalent circuit of Type-III model with different switch-off timing of S3. (b) Transistor-level simulation results on the effect of switch-off timing of S3.

It is worth noting that the variation of delay mismatch between clocks driving S1 and S2 also introduces variable differential charge injection while the variation is in the range of $\Delta T_{V P 2}$ (similar to $\Delta T_{V P 1}$ in Type-II model in Fig. $7(\mathrm{~b})$ ), as shown in Fig. 9(b). This is because the resistance of $R_{S 3}$ is not infinitesimal and very small coupling effect still exists. The solution is similar to that in Type-II model, i.e., the time offset $\Delta T_{\text {OFFSET }}$ between the clocks driving $\mathrm{S} 1$ and $\mathrm{S} 2$ is set to a value larger than $\Delta T_{V P 2}$ in order to minimize the variation of charge injection.

Based on the above analysis, a clock scheme for practical capacitive half-bridges is proposed as shown in Fig. 10. In the scheme, three multi-nested clocks are needed to minimize the variation of charge injection, which turn off switches in the following sequence. The switches connected to the differential node $A$ are turned off by the clock $\Phi 1 n^{2}$ first, and then the switches connected to the differential node B are turned off by the clock $\Phi 1 \mathrm{n}$, finally the switches connected to the common node are turned off by the clock $\Phi 1$.

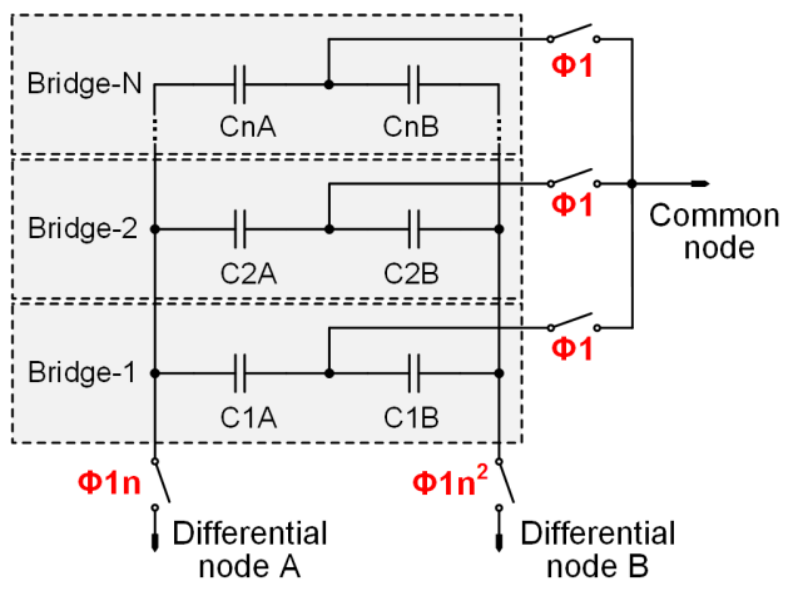

Fig. 10. Clock strategy to minimize the differential charge injection for multi-capacitive-bridge.

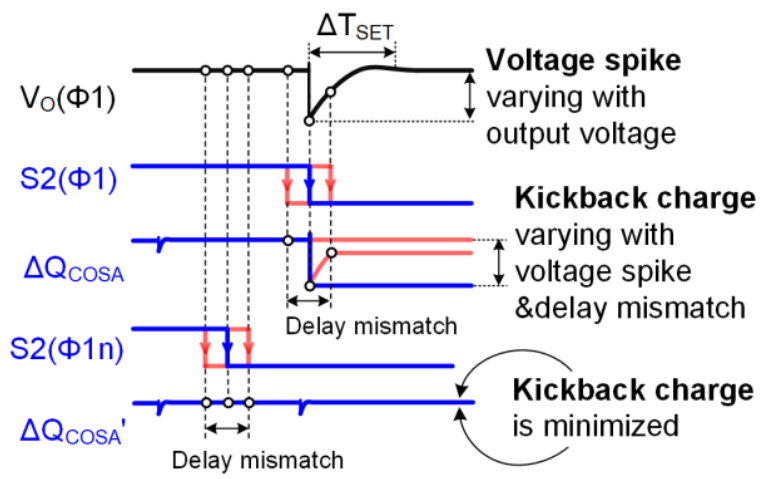

Fig. 11. Clock strategy to eliminate the kickback charge injection for capacitors in the feedback network.

\section{Analysis of Type-IV model}

The main consideration of Type-IV model shown in Fig. 4(d) is the kickback charge injection introduced by output voltage spike. It is well known that kickback noise in ADC is caused by the high-speed turnover of the regeneration comparator's output and it introduces reference source noise, code transition error and settling time degradation of amplifiers [26]. Similarly, in this work, the "kickback charge injection" is caused by the voltage spike of the readout circuit's output and it introduces both nonlinearity and input offset variation. The process of injection of kickback charge is described as follows.

Firstly, the voltage spike is caused by the charge injected from the switches connected to the output terminals, e.g., the switch S3 driven by phase $\Phi 1$ in Fig. 4(d). The voltage spike takes a time period $\Delta T_{S E T}$ to settle, as shown in Fig.11. $\Delta T_{S E T}$ depends on the gain-bandwidth product $\left(g_{m} / C_{0}\right)$ of the amplifier. Then, as the switch $\mathrm{S} 2$ driven by the same phase $\Phi 1$ in Fig. 4(d) will turned off within the period $\Delta T_{S E T}$ due to delay mismatch, the voltage spike injects an error charge $\Delta Q_{\operatorname{CoSA}}$ to the capacitor $C_{O S A}$. Finally, the error charge $\Delta Q_{\operatorname{COSA}}$ is transferred to the integration capacitor $C_{i}$ in the phase $\Phi 2$ (Fig. 1), producing output error.

Based on the above analysis, the output error due to kick back charge from the switch S3 varies with the change of delay mismatch between the switch $\mathrm{S} 2$ and the switch $\mathrm{S} 3$. This results in variation of input offset. Besides, the amount of kick back charge injected from the switch S3 varies with its bias voltage 
which is equal to the output voltage $V_{O}$. This results in nonlinear output error. Thus the kick back charge injection introduces both nonlinearity and input offset variation.

The kickback charge injection can be minimized by driving the switch S2 with an earlier clock $(\Phi 1 \mathrm{n})$ than that driving the switch S3 (Ф1) in order to minimize the variation of kickback charge $\Delta Q_{\operatorname{COSA}}$, as shown in Fig. 11.

\section{PHYSICAL VERIFICATION}

To apply the multi-nested clocks, the OSA readout circuit from [7] is decomposed into the four types of models described in Section III, as shown in Fig. 12. All the switches used in the readout circuit are the LSC switches. The timing diagram of the clocks is shown in Fig. 8 with $\Delta T_{\text {OFFSET }}$ being 10ns. The operational frequency is $100 \mathrm{kHz}$.

The OSA readout circuit from [7] is reproduced without changing circuit structure and the proposed multi-nested clocks are demonstrated in the reproduced circuit. The test configuration is shown in Fig. 13. Both the multi-nested clocks and the traditional clocks are generated on chip. Compared with the traditional clock generator, the chip area and the current supply of the multi-nested clock generator are increased by $125 \%$ (from $2,000 \mu \mathrm{m}^{2}$ to $4,500 \mu \mathrm{m}^{2}$ ) and $113 \%$ (from $80 \mathrm{nA}$ to $170 \mathrm{nA}$ at $100 \mathrm{kHz}$ operation frequency), respectively. However, the additional area and power consumption introduced by the multi-nested clock generator are negligible in terms of the overall chip area and power consumption. A multiplexer controlled by off-chip signal is used to select clock. An on-chip test module is added to mimic the input signal charge of a sensor. The module is composed of the capacitors $C_{T}$ and relevant switches. The capacitance of $C_{T}$ is $20 \mathrm{fF}$, which means that with an input test voltage $V_{T}$ of $45 \mathrm{mV}$, it can mimic $0.9 \mathrm{fC}$ input signal charge which is equal to the amount of signal charge produced by a sensing element's capacitance difference of $1.0 \mathrm{fF}$. The readout circuit is fabricated with a commercial $1.8 \mathrm{~V} 0.18 \mu \mathrm{m}$ BCD process. The photograph of the chip is shown in Fig. 14.

The test results of the readout circuit are shown in Fig. 15. The main experimental parameters are listed in Table I. The number of testing samples of the chip is 15 . For the OSA readout circuit without the multi-nested clocks, the average equivalent input offset of the readout circuit is $\mathrm{OS} 1=1.66 \mathrm{fF}$. The offset variation is $\delta 1=1.4 \mathrm{fF}$ (from $0.9 \mathrm{fF}$ to $2.3 \mathrm{fF}$ ). The large offset variation indicates that the readout circuit is sensitive to the variation of fabrication process and this is due to the charge injection variation caused by the timing mismatch discussed in Section III. For the OSA readout circuit with the multi-nested clocks, the average equivalent input offset of the readout circuit is reduced from $1.66 \mathrm{fF}$ to $0.25 \mathrm{fF}(\mathrm{OS} 2)$ and the offset variation is reduced from $1.4 \mathrm{fF}$ to $0.2 \mathrm{fF}$ ( $\delta 2$, from $0.1 \mathrm{fF}$ to $0.3 \mathrm{fF}$ ). The nonlinearity of the readout circuit is also significantly reduced from $5.5 \%$ to $0.9 \%$ with an input signal range of 30fF. This is because the kickback charge injection is reduced. These measurement results demonstrate that the multi-nested clocks can significantly reduce the charge injection and charge injection variation introduced by the

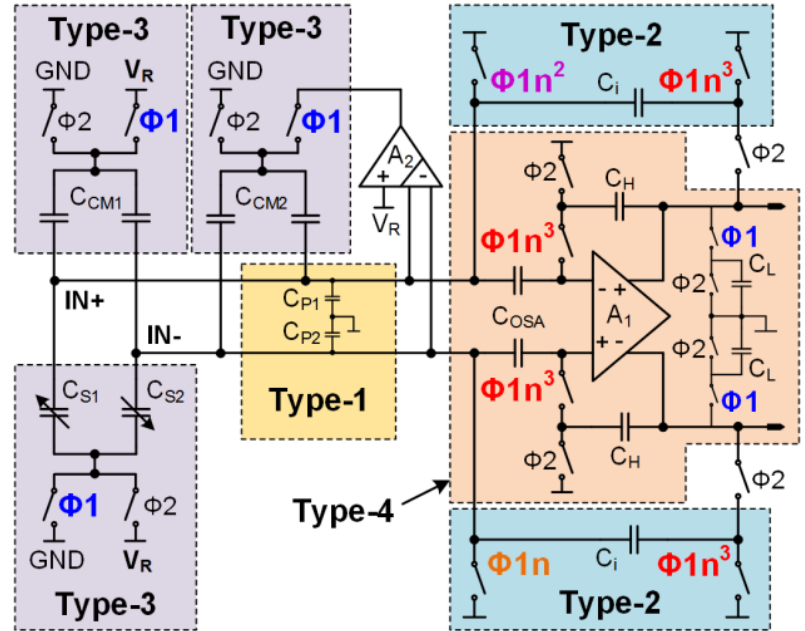

Fig. 12. Decomposition of the OSA readout circuit and the clock strategy employing multi-nested clocks.

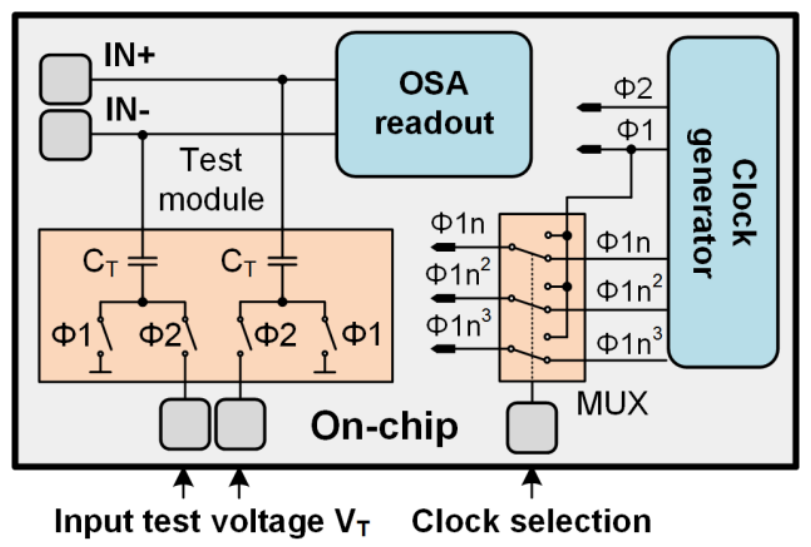

Fig. 13. Test configuration of the readout circuit.

timing mismatch. Compared to [14] and [16] which were fabricated with the same IC process and reduced their input offset to sub-fF level by calibration method, this work consumes significantly less chip area. This is because this work does not require the calibration circuits used in [14] and [16] which consume a high proportion of their chip area. The reference [14] achieved a marginally better input offset (0.224fF), compared to this work, but it has more than doubled chip area $\left(1.13 \mathrm{~mm}^{2}\right)$. Furthermore, the calibration circuits used in the [14] and [16] do not take measures to reduce the nonlinearity while this work does. The final nonlinearity of this work $(0.9 \%)$ is slight worse than that of [16] $(0.8 \%)$. This is because the supply voltage used in this work $(1.8 \mathrm{~V})$ is much lower than that in [16] (3.3V).

\section{CONCLUSIONS}

In order to improve the precision of the readout circuit for capacitive sensor, a clock strategy using the multi-nested clocks is proposed and applied to a power-efficient OSA readout circuit in this paper. Compared to the OSA readout circuit employing a traditional clock scheme [7], the OSA readout circuit using the multi-nested clocks reduces the equivalent input offset from $1.66 \mathrm{fF}$ to $0.25 \mathrm{fF}$ and the offset variation from $1.4 \mathrm{fF}$ to $0.2 \mathrm{fF}$. The nonlinearity measured by the output error of 


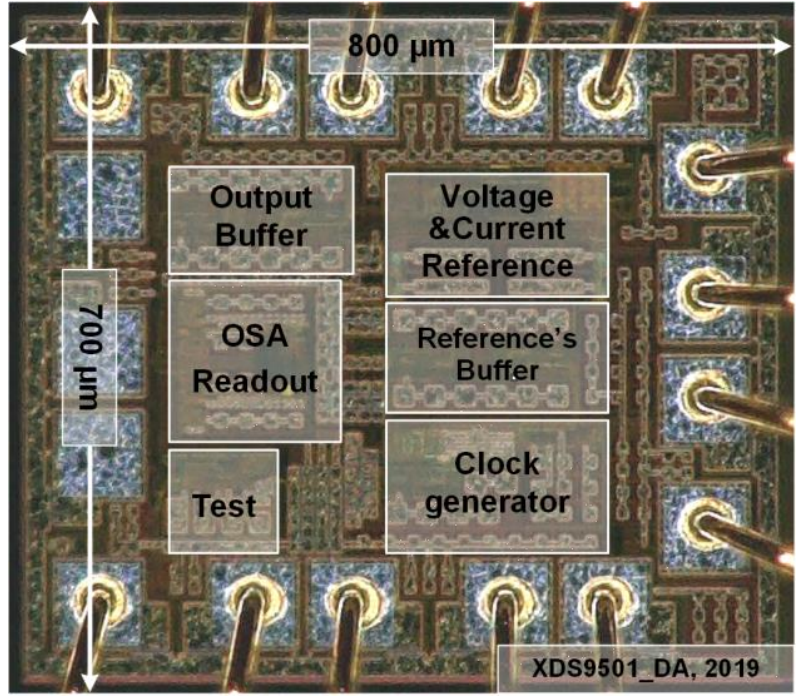

Fig. 14. Chip photograph of the readout circuit.

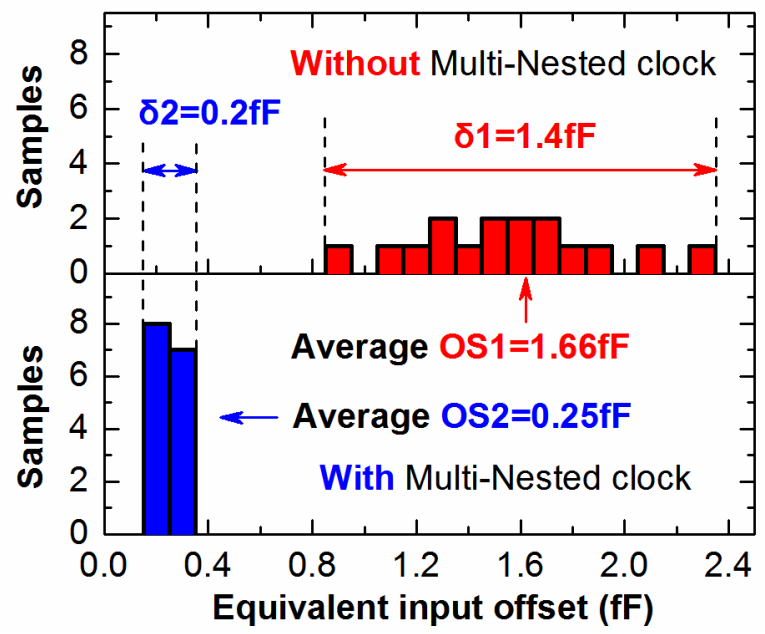

Fig. 15. Measurement results of the output signal of the readout circuit with and without multi-nested clocks, respectively.

TABLE I MAIN PARAMETERS SUMMARY AND COMPARISONS

\begin{tabular}{ccccc}
\hline \hline & This work & {$[7]$} & {$[14]$} & {$[16]$} \\
\hline Equ. input offset $(\mathrm{fF})$ & $\mathbf{0 . 2 5}$ & $\mathbf{1 . 6 6}$ & $\mathbf{0 . 2 2 4}$ & $\mathbf{0 . 4 8 8}$ \\
Offset deviation $(\mathrm{fF})$ & $\mathbf{0 . 2}$ & $\mathbf{1 . 4}$ & - & - \\
Non-linearity & $\mathbf{0 . 9 \%}$ & $\mathbf{5 . 5 \%}$ & - & $\mathbf{0 . 8 \%}$ \\
Chip area $\left(\mathrm{mm}^{2}\right)$ & $\mathbf{0 . 5 5}$ & $\mathbf{0 . 5 5}$ & $\mathbf{1 . 1 3 *}$ & $\mathbf{1 . 0 2}$ \\
IC Process $(\mu \mathrm{m})$ & 0.18 & 0.18 & 0.18 & 0.18 \\
Full input range $(\mathrm{fF})$ & \pm 30 & \pm 30 & - & \pm 150 \\
CVC sensitivity $(\mathrm{mV} / \mathrm{fF})$ & 45 & 45 & 2 & 9.5 \\
Supply voltage $(\mathrm{V})$ & 1.8 & 1.8 & 3.3 & 3.3 \\
Power $(\mu \mathrm{W})$ & 451 & 450 & 980 & 220 \\
Input noise $(\mathrm{aF} / \sqrt{ } \mathrm{Hz})$ & 1.9 & 1.9 & 0.088 & 1.275 \\
Bandwidth $(\mathrm{kHz})$ & 10 & 10 & 0.4 & 0.4 \\
\hline \hline
\end{tabular}

*Data is estimated from the chip photograph excluding ADC which does not appear in this work in order to give a fair comparison.

the readout circuit with an input signal range of $30 \mathrm{fF}$ is reduced from $5.5 \%$ to $0.9 \%$. Compared to another similar work [16] which reduced the offset of readout circuit to $0.488 \mathrm{fF}$ by the calibration method, this work achieves a better result with the offset of $0.25 \mathrm{fF}$.

\section{REFERENCES}

[1] Althoff, Tim, et al. "Large-scale physical activity data reveal worldwide activity inequality." Nature, vol. 547, no. 7663, pp.336-339, Jul. 2017.

[2] Balducci S, et al. "Effect of a Behavioral Intervention Strategy for Adoption and Maintenance of a Physically Active Lifestyle: The Italian Diabetes and Exercise Study (IDES) 2: A Randomized Controlled Trial." Diabetes Care, vol. 40, no.11, pp. 1444-1452, Nov. 2017.

[3] Lyall L M, et al. "Association of disrupted circadian rhythmicity with mood disorders, subjective wellbeing, and cognitive function: a cross-sectional study of 91105 participants from the UK Biobank." Lancet Psychiatry, vol. 5, no.6, pp. 507-514, Jun. 2018.

[4] J. Reynolds, P. Ahmmed and A. Bozkurt, "An Injectable System for Subcutaneous Photoplethysmography, Accelerometry, and Thermometry in Animals," IEEE Trans. Biomed. Circuits Syst.. Early access. 2019. doi: 10.1109/TBCAS.2019.2923153

[5] A. D. Wiens and O. T. Inan, "A Novel System Identification Technique for Improved Wearable Hemodynamics Assessment," IEEE Trans. Biomed. Eng., vol. 62, no. 5, pp. 1345-1354, May 2015.

[6] V. P. Petkov, G. K. Balachandran and J. Beintner, "A Fully Differential Charge-Balanced Accelerometer for Electronic Stability Control," IEEE J. Solid-State Circuits, vol. 49, no. 1, pp. 262-270, Jan. 2014.

[7] L. Zhong, X. Lai and D. Xu, "Oversampling Successive Approximation Technique for MEMS Differential Capacitive Sensor," IEEE J. Solid-State Circuits, vol. 53, no. 8, pp. 2240-2251, May. 2018.

[8] L. Zhong, et al, "Differential Capacitive Readout Circuit Using Oversampling Successive Approximation Technique," IEEE Trans. Circuits Syst. I, Reg. Papers. vol. 65, no. 12, pp. 4072-4085, Dec. 2018.

[9] J. Zhao, et al., "A System Decomposition Model for Phase Noise in Silicon Oscillating Accelerometers," IEEE Sensors J., vol. 16, no. 13, pp. 5259-5269, July1, 2016.

[10] J. Zhao et al., "A 0.23- g Bias Instability and 1-ug $/ \sqrt{ } \mathrm{Hz}$ Acceleration Noise Density Silicon Oscillating Accelerometer With Embedded Frequency-to-Digital Converter in PLL," IEEE J. Solid-State Circuits, vol. 52, no. 4, pp. 1053-1065, April 2017.

[11] Xu H, et al. "A Closed-Loop $\Sigma \Delta$ Interface for a High-Q Micromechanical Capacitive Accelerometer With $200 \mathrm{ng} / \sqrt{ } \mathrm{Hz}$ Input Noise Density", IEEE J. Solid-State Circuits, vol.50, no.9, pp.2101-2112, 2015.

[12] Amini B. V., Ayazi F., "A 2.5-V 14-bit $\Sigma \Delta$ CMOS SOI capacitive accelerometer," IEEE J. Solid-State Circuits, vol. 39, no. 12, pp. 2467-2476, 2004.

[13] Enz C. C, Temes G. C., "Circuit techniques for reducing the effects of op-amp imperfections: autozeroing, correlated double sampling, and chopper stabilization," Proceedings of the IEEE, 1996, 84(11):1584-1614.

[14] Mun Y., Kim H , Ko Y., et al. "Capacitive analog front-end circuit with dual-mode automatic parasitic cancellation loop," Microsyst Technol, 2017, 23(2):515-523.

[15] H. K. Ouh et al., "Sub-fF trimmable readout circuit for tri-axes capacitive microaccelerometers," SENSORS, 2012 IEEE, Taipei, 2012, pp. 1-4.

[16] Haryong S, Yunjong P, Hyungseup K, et al. "Fully Integrated Low-Noise Readout Circuit with Automatic Offset Cancellation Loop for Capacitive Microsensors," Sensors, vol. 15, no. 10, pp. 26009-26017, Oct.2015.

[17] G. Wegmann, E. A. Vittoz and F. Rahali, "Charge injection in analog MOS switches," IEEE J. Solid-State Circuits, vol. 22, no. 6, pp. 1091-1097, Dec. 1987.

[18] B. R. Schiffer, A. Burstein and W. J. Kaiser, "An active charge cancellation system for switched-capacitor sensor interface circuits," IEEE J. Solid-State Circuits, vol. 33, no. 12, pp. 2134-2138, Dec. 1998.

[19] G. T. Ong and P. K. Chan, "A Power-Aware Chopper-Stabilized Instrumentation Amplifier for Resistive Wheatstone Bridge Sensors," IEEE Trans. Instrum. Meas., vol. 63, no. 9, pp. 2253-2263, Sept. 2014.

[20] J. Xu, Q. Fan, J. H. Huijsing, C. Van Hoof, R. F. Yazicioglu and K. A. A. Makinwa, "Measurement and Analysis of Current Noise in Chopper Amplifiers," IEEE J. Solid-State Circuits, vol. 48, no. 7, pp. 1575-1584, July 2013

[21] P. Vejdani and F. Nabki, "Dual-Path and Dual-Chopper Amplifier Signal Conditioning Circuit with Improved SNR and Ultra-Low Power Consumption for MEMS," IEEE Trans. Circuits Syst. I, Reg. Papers, vol. 66, no. 6, pp. 2253-2262, June 2019.

[22] O. Choksi and L. R. Carley, "Analysis of switched-capacitor common-mode feedback circuit," IEEE Transactions on Circuits and Systems II: Analog and Digital Signal Processing, vol. 50, no. 12, pp. 906-917, Dec. 2003. 
[23] M. Schipani, P. Bruschi, G. C. Tripoli and T. Ungaretti, "A low power CMOS interface circuit for three-axis integrated accelerometers," 2007 Ph.D Research in Microelectronics and Electronics Conference, Bordeaux, 2007, pp. 117-120.

[24] Frangi A, De Masi B, Simoni B. "Microelectromechanical three-axis capacitive accelerometer," U.S. Patent 8863575 B2, 2014.

[25] M. Lemkin and B. E. Boser, "A three-axis micromachined accelerometer with a CMOS position-sense interface and digital offset-trim electronics," IEEE J. Solid-State Circuits, vol. 34, no. 4, pp. 456-468, Apr. 1999.

[26] P. M. Figueiredo and J. C. Vital, "Kickback noise reduction techniques for CMOS latched comparators," IEEE Trans. Circuits Syst., II, Exp. Briefs, vol. 53, no. 7, pp. 541-545, July 2006.

[27] B. Murmann, "Thermal noise in track-and-hold circuits: Analysis and simulation techniques," IEEE Solid-State Circuits Mag., vol. 4, no. 2, pp. 46-54, Jun. 2012. 